\title{
Efficacy and Safety of Alemtuzumab Through 9 Years of Follow-up in Patients with Highly Active Disease: Post Hoc Analysis of CARE-MS I and II Patients in the TOPAZ Extension Study
}

\author{
Tjalf Ziemssen ${ }^{1}$ (D) Ann D. Bass ${ }^{2} \cdot$ Regina Berkovich $^{3,4} \cdot$ Giancarlo Comi $^{5}$. Sara Eichau ${ }^{6}$. Jeremy Hobart ${ }^{7}$. \\ Samuel F Hunter ${ }^{8}$. Christopher LaGanke ${ }^{9}$. Volker Limmroth ${ }^{10}$. Daniel Pelletier ${ }^{4}$. Carlo Pozzilli ${ }^{11}$. \\ Sven Schippling ${ }^{12} \cdot$ Livia Sousa $^{13} \cdot$ Anthony Traboulsee $^{14} \cdot$ Bernard M. J. Uitdehaag ${ }^{15}$. Bart Van Wijmeersch ${ }^{16}$. \\ Zia Choudhry ${ }^{17}$. Nadia Daizadeh ${ }^{17}$. Barry A. Singer ${ }^{18}$ on behalf of CARE-MS I, CARE-MS II, CAMMS03409, and \\ TOPAZ investigators
}

Published online: 24 July 2020

(c) The Author(s) 2020

\begin{abstract}
Background Alemtuzumab efficacy versus subcutaneous interferon- $\beta$-1a (SC IFNB-1a) was demonstrated over 2 years in patients with relapsing-remitting multiple sclerosis, with continued efficacy over 7 additional years. Alemtuzumab is included as a recommended treatment for patients with highly active disease (HAD) by the American Academy of Neurology Practice Guidelines, and the label indication in Europe was recently restricted to the treatment of HAD patients. There is currently no consensus definition for HAD, and alemtuzumab efficacy across various HAD definitions has not been explored previously. Objectives In this post hoc analysis, we assess the efficacy and safety of alemtuzumab in Comparison of Alemtuzumab and Rebif ${ }^{\circledR}$ Efficacy in Multiple Sclerosis (CARE-MS) trial patients who met criteria for at least one of four separate definitions of HAD (one primary and three alternatives). Over 2 years, alemtuzumab-treated HAD patients were compared with SC IFNB-1a-treated HAD patients, with additional 7-year follow-up in patients from the alemtuzumab arm.

Methods Patients in the CARE-MS studies received either alemtuzumab (baseline: 5 days; 12 months later: 3 days) or SC IFNB-1a (3 times weekly). Alemtuzumab-treated patients who enrolled in the extensions could receive additional courses $\geq 12$ months apart. Four definitions of HAD were applied to assess alemtuzumab efficacy: the pre-specified primary definition (two or more relapses in the year prior to baseline and at least one gadolinium [Gd]-enhancing lesion at baseline) and three alternative definitions that focused on relapse, magnetic resonance imaging (MRI), or prior treatment response criteria. Efficacy outcomes were annualized relapse rate, change in Expanded Disability Status Scale score, 6-month confirmed disability worsening, 6-month confirmed disability improvement, MRI disease activity, and brain volume change. Adverse events were summarized for HAD patients meeting the primary definition.

Results In the pooled CARE-MS population, 208 alemtuzumab-treated patients met the primary HAD definition. Annualized relapse rate was 0.27 in years $0-2$ and 0.16 in years 3-9. Over 9 years, $62 \%$ of patients were free of 6-month confirmed disability worsening, 50\% had 6-month confirmed disability improvement, and median cumulative change in brain volume was $-2.15 \%$. During year $9,62 \%$ had no evidence of disease activity, and $69 \%$ were free of MRI disease activity. Similar efficacy outcomes were observed using an alternative relapse-driven HAD definition. For patients meeting alternative HAD definitions focused on either higher MRI lesion counts or disease activity while on prior therapy, reduced efficacy for some endpoints was seen. Safety was consistent with the overall CARE-MS population through year 9.

Conclusions Over 9 years, alemtuzumab efficacy was maintained in CARE-MS HAD patients based on four HAD definitions. These results support intervention with alemtuzumab in patients with early indicators of HAD, including frequent relapse without high MRI activity. No safety signals were observed over 9 years that were unique to the HAD populations. ClinicalTrials.gov Identifiers NCT00530348; NCT00548405; NCT00930553; NCT02255656.
\end{abstract}

Electronic Supplementary Material The online version of this article (https://doi.org/10.1007/s40263-020-00749-x) contains supplementary material, which is available to authorized users.

Extended author information available on the last page of the article 


\section{Key Points}

Using four definitions of highly active disease (HAD) with varying criteria, alemtuzumab improved outcomes in patients with HAD, and efficacy was maintained over 9 years in highly active relapsing-remitting multiple sclerosis.

Efficacy in the HAD population was generally consistent with and similar to the overall Comparison of Alemtuzumab and Rebif ${ }^{\circledR}$ Efficacy in Multiple Sclerosis (CARE-MS) population.

No new safety signals were seen in alemtuzumab-treated patients with HAD over 9 years.

\section{Introduction}

In patients with relapsing-remitting multiple sclerosis (RRMS), highly active disease (HAD) is associated with a more aggressive disease course, leading to an accelerated rate of disease progression [1]. Generally, HAD has been defined as rapid accumulation of disability, frequent relapses, and high magnetic resonance imaging (MRI) activity. Criteria vary, however, with some defining HAD using Expanded Disability Status Scale (EDSS) score and response to treatment along with relapse and MRI activity [2-4]. Efforts to refine the definition of HAD have been complicated by the potential to be either too restrictive (i.e., inadvertently eliminating patients) or too lax (i.e., consequently including patients without need for aggressive treatment) [2]. Nonetheless, effective early treatment would be expected to limit progression and disability. Some moderate- to high-efficacy disease-modifying therapies (DMTs) are already indicated for HAD [5-8], and recent updates to treatment guidelines for the American Academy of Neurology and the European label include alemtuzumab as an option for HAD patients $[9,10]$.

In the phase 3 Comparison of Alemtuzumab and Rebif ${ }^{\circledR}$ Efficacy in Multiple Sclerosis (CARE-MS) studies (NCT00530348; NCT00548405), alemtuzumab improved clinical and MRI outcomes versus subcutaneous interferon$\beta-1 \mathrm{a}$ (SC IFNB-1a) in patients with active RRMS [11, 12]. Efficacy was maintained over an additional 7 years in two consecutive extension studies (CARE-MS extension [NCT00930553] and the ongoing TOPAZ study [a long-Term follow-up study for multiple sclerOsis Patients who have completed the AlemtuZumab extension study; NCT02255656]) [13-16]. Adverse events (AEs) associated with alemtuzumab treatment in clinical trials and postmarketing experience include infusion-associated reactions (IARs), increased frequency of infection and the potential for opportunistic infections, secondary autoimmunity (thyroid disorders, immune thrombocytopenia [ITP], nephropathies, autoimmune cytopenias, autoimmune hepatitis, and other less common autoimmune events), acute acalculous cholecystitis, and cardiovascular and pulmonary events possibly related to infusion $[9,11-13,15,17-21]$.

In this post hoc analysis, we assess the efficacy and safety of alemtuzumab in CARE-MS patients who met criteria for at least one of four separate definitions of HAD (one primary and three alternatives) with the intent to cover patients who are variably defined as having HAD. Over 2 years, alemtuzumab-treated HAD patients were compared with SC IFNB1a-treated HAD patients, with an additional 7-year follow-up in patients from the alemtuzumab arm.

\section{Methods}

\subsection{Design of CARE-MS Core Studies and Extension Studies}

The CARE-MS core studies and their extensions have been described previously $[11,12]$. Briefly, the 2-year, core phase 3 CARE-MS studies compared alemtuzumab with SC IFNB$1 \mathrm{a}$ in patients with active RRMS who were either treatment-naive (CARE-MS I) or had an inadequate response to prior therapy (CARE-MS II) $[11,12]$. In the core studies, patients received either alemtuzumab $12 \mathrm{mg} /$ day intravenous (baseline: 5 consecutive days; 12 months later: 3 consecutive days), or SC IFNB-1a $44 \mu \mathrm{g}$ (3 times per week) $[11,12]$. Alemtuzumab-treated patients who completed the core studies and entered the 4-year CARE-MS extension could receive additional, as-needed alemtuzumab (12 mg/ day; 3 consecutive days $\geq 12$ months apart) for relapse or MRI activity, or receive other licensed DMTs per investigator's discretion $[13,15]$. SC IFNB-1a-treated patients could also enter the extension and receive alemtuzumab; however, outcomes for these patients beyond year 2 are not presented because SC IFNB-1a treatment was discontinued thereafter. Follow-up of patients beyond 6 years is continuing in the TOPAZ extension, wherein patients can receive additional alemtuzumab courses ( $12 \mathrm{mg} /$ day; 3 consecutive days $\geq 12$ months apart) or other DMT at any time, both per investigator's discretion (no criteria) $[14,16]$.

\subsection{Clinical Efficacy Assessments}

Relapses were defined as new neurologic symptoms attributable to multiple sclerosis (MS) lasting $\geq 48 \mathrm{~h}$, with an 
objective change in neurologic examination; annualized relapse rate (ARR) and proportions of patients free of relapse were reported. EDSS score was assessed quarterly and at the time of suspected relapse by raters who were blinded throughout the follow-up period to core study treatment assignment and treatment history. Changes in EDSS score were defined as improved ( $\geq 1.0$-point decrease from core study baseline), stable ( $\leq 0.5$-point change in either direction from core study baseline), and worsened ( $\geq 1.0$ point increase from core study baseline). Confirmed disability worsening (CDW) was defined as a $\geq 1.0$-point EDSS score increase (or $\geq 1.5$ points if baseline EDSS $=0$ ) confirmed over 6 months. Confirmed disability improvement (CDI) was defined as a $\geq 1.0$-point EDSS score decrease from baseline confirmed over 6 months (assessed only in patients with baseline EDSS score $\geq 2.0$ ). MRI was assessed annually by imaging specialists blinded to core study treatment assignment and treatment history. Freedom from MRI disease activity was defined as no new gadolinium (Gd)enhancing T1 lesions on current MRI and no new/enlarging T2 hyperintense lesions since last MRI. No evidence of disease activity (NEDA) was defined as the absence of relapse, 6-month CDW, and MRI disease activity. Brain volume loss (BVL) was assessed by brain parenchymal fraction (BPF); blinded scans were read at the Cleveland Clinic (Cleveland, $\mathrm{OH}, \mathrm{USA})$.

\subsection{Safety Monitoring}

Safety monitoring occurred for $\geq 48$ months following the last alemtuzumab administration, according to the recommended risk-minimization protocol, which included hematology (complete blood counts with differential; at least monthly), renal examinations (serum creatinine and urinalysis with microscopy; monthly), and thyroid function (at least quarterly). All AEs, serious AEs, and medical events of interest were recorded. IARs were defined as any AE with onset during or $\leq 24 \mathrm{~h}$ after the end of infusion.

\subsection{HAD Definitions and Analyses}

Four different definitions of HAD were applied to the core CARE-MS I and II populations (Fig. 1a), resulting in four groups of HAD patients (i.e., primary definition and alternative definitions $1-3$ ). Some patients met more than one HAD definition and were, therefore, included in more than one group.

The primary HAD definition was pre-specified in the CARE-MS I and II trial protocols, and was defined as patients having two or more relapses in the year prior to baseline and at least one Gd-enhancing lesion at baseline (Fig. 1a), and is one of the most used in the literature [6, $8,22,23]$. Outcomes from the CARE-MS I and II studies individually, as well as the pooled CARE-MS studies, were assessed for those fitting this primary HAD definition.

Three alternative HAD definitions (Fig. 1a) were also applied to the pooled population from the CARE-MS studies, and sensitivity analyses of efficacy outcomes were assessed for the subgroups fitting each alternative definition. The first alternative HAD definition focused on relapse; patients met the criteria for the first definition if they had two or more relapses in the year prior to core study baseline, independent of baseline Gd-enhancing lesion count. The second alternative HAD definition focused on MRI lesions; patients met the criteria for the second definition if they had at least one relapse in the year prior and three or more Gd-enhancing lesions at core study baseline. The third alternative HAD definition focused on prior therapy failure; patients met the criteria for the third definition if they had at least one relapse and at least one Gd-enhancing lesion in the year prior to baseline while on therapy with another DMT. Because all CARE-MS I patients were treatment-naive at core study baseline, none met the third alternative definition.

\subsection{Statistical Analyses}

The statistical analyses for alemtuzumab were based on all available interim data through year 9 (TOPAZ year 3); data cut-off date was 20 October 2018. ARR was estimated using a negative binomial model with robust variance estimation. Mean EDSS scores from core study baseline through year 9 were evaluated. Ranked analysis of covariance (ANCOVA) with adjustment for geographic region and baseline EDSS score was used to compare changes in EDSS scores from baseline through year 2 in alemtuzumab-treated versus SC IFNB-1a-treated patients. Proportions of patients free of 6-month CDW or achieving 6-month CDI were assessed using Kaplan-Meier estimates. Proportions of patients free of MRI disease activity, new Gd-enhancing T1 lesions, new/ enlarging $\mathrm{T} 2$ hyperintense lesions, and new $\mathrm{T} 1$ hypointense lesions were analyzed using logistic regression with covariate adjustment for baseline values; 95\% confidence intervals (CIs) were obtained by normal approximation to the binomial distribution. Percentage change in BPF from core study baseline was evaluated at each time point; distribution-free estimates were obtained for the CI of the median. All analyses were carried out using SAS statistical software (version 9.4, The SAS Institute, Cary, NC).

\subsection{Standard Protocol Approvals, Registrations, and Patient Consents}

CARE-MS I, CARE-MS II, CAMMS03409, and TOPAZ are registered with ClinicalTrials.gov (NCT00530348; NCT00548405; NCT00930553; NCT02255656). Patients provided written informed consent, and all procedures were 


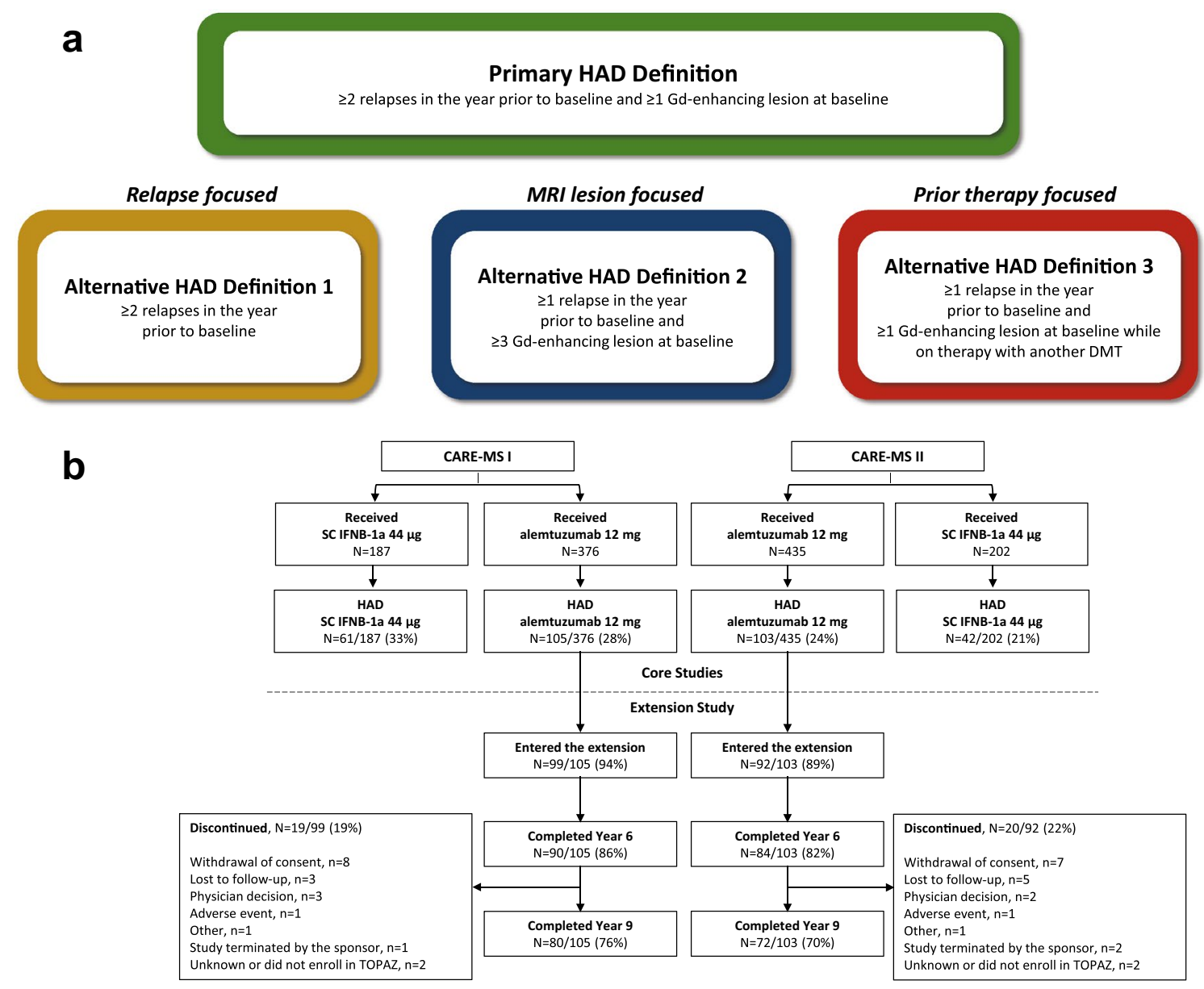

Fig. 1 HAD definitions and HAD patient disposition: a primary and alternative definitions of HAD used in this post hoc analysis; $\mathbf{b}$ CARE-MS I and II alemtuzumab-treated HAD patient disposition through year 9 defined by the primary HAD definition. CARE-MS

approved by local institutional ethics review boards of participating sites.

\section{Results}

\subsection{Patients and Baseline Characteristics: Primary HAD Definition}

At core study baseline, $28 \%$ of CARE-MS I and $24 \%$ of CARE-MS II alemtuzumab-treated patients met the primary HAD definition; of these, 70-76\% remained in the study through year 9 (Fig. 1b). These retention rates were similar to those in the overall CARE-MS populations $(66-75 \%)$ and in the CARE-MS patients without HAD (65-75\%). Total follow-up time over 9 years in CARE-MS alemtuzumab-treated HAD patients is presented in Supplementary Table 1 (see the electronic supplementary material). Over years $3-9,56 \%$ of CARE-MS I and $43 \%$ of CARE-MS II alemtuzumab-treated
Comparison of Alemtuzumab and Rebif ${ }^{\circledR}$ Efficacy in Multiple Sclerosis, $D M T$ disease-modifying therapy, $G d$ gadolinium, $H A D$ highly active disease, MRI magnetic resonance imaging, SC IFNB-1 $a$ subcutaneous interferon- $\beta$ - $1 \mathrm{a}$

HAD patients received neither additional alemtuzumab nor another DMT, comparable to $55 \%$ and $41 \%$ of patients in the overall CARE-MS I and II populations, respectively.

At baseline, HAD patients were slightly younger, with shorter disease duration, more prior relapses, higher T2 hyperintense lesion volumes, and more Gd-enhancing lesions when compared with the overall CARE-MS population; baseline T2 hyperintense lesion counts were not available. More CARE-MS II patients with HAD received two or more previous DMTs compared with the overall CAREMS II population (Table 1). Baseline characteristics for the overall CARE-MS I and II populations have been published previously [11, 12]

\subsection{Efficacy: Primary HAD Definition}

In HAD patients from CARE-MS I and II, ARR over years $0-2$ was significantly reduced with alemtuzumab versus SC IFNB-1a $(p \leq 0.01)$ and remained low over years 3-9, 
Table 1 Baseline characteristics for the HAD alemtuzumab-treated patients in CARE-MS I and II

\begin{tabular}{|c|c|c|}
\hline \multirow[t]{2}{*}{ Baseline characteristic } & \multicolumn{2}{|c|}{ Alemtuzumab-treated HAD patients } \\
\hline & CARE-MS I $(N=105)$ & $\begin{array}{l}\text { CARE-MS } \\
\text { II }(N=103)\end{array}$ \\
\hline Age (years) & $32.1(8.0)$ & $32.7(7.7)$ \\
\hline Female, $n(\%)$ & $69(66)$ & $69(67)$ \\
\hline EDSS score & $2.0(0.8)$ & $2.6(1.2)$ \\
\hline Years since initial relapse & $1.7(1.4)$ & $4.0(2.6)$ \\
\hline Number of relapses in prior 1 year & $2.3(0.6)$ & $2.4(0.8)$ \\
\hline Number of relapses in prior 2 years & $2.9(1.0)$ & $3.4(1.4)$ \\
\hline Gd-enhancing lesion count & $5.6(7.1)$ & $5.2(6.2)$ \\
\hline Patients with Gd-enhancing lesions, $n(\%)$ & $105(100)$ & $103(100)$ \\
\hline T2-hyperintense lesion volume $\left(\mathrm{cm}^{3}\right)$ & $9.9(10.1)$ & $12.1(12.2)$ \\
\hline Brain parenchymal fraction & $0.82(0.02)$ & $0.82(0.02)$ \\
\hline \multicolumn{3}{|l|}{ Number of previous DMTs received, $n(\%)$} \\
\hline 0 & $105(100)$ & 0 \\
\hline 1 & - & $69(67)$ \\
\hline 2 & - & $26(25)$ \\
\hline 3 & - & $5(5)$ \\
\hline$\geq 4$ & - & $3(3)$ \\
\hline \multicolumn{3}{|l|}{ Previous DMTs received, $n(\%)$} \\
\hline IFNB-1a & - & $59(57)$ \\
\hline IFNB-1b & - & $32(31)$ \\
\hline Glatiramer acetate & - & $42(41)$ \\
\hline Natalizumab & - & $7(7)$ \\
\hline Immunoglobulin & - & $3(3)$ \\
\hline Azathioprine & - & $4(4)$ \\
\hline
\end{tabular}

Values are mean (SD) unless otherwise noted

Primary HAD definition: $\geq 2$ relapses in the year prior to baseline and $\geq 1$ Gd-enhancing lesion at baseline

CARE-MS Comparison of Alemtuzumab and Rebif ${ }^{\circledR}$ Efficacy in Multiple Sclerosis, DMT disease-modifying therapy, EDSS Expanded Disability Status Scale, $G d$ gadolinium, $H A D$ highly active disease, IFNB interferon- $\beta$; $S D$ standard deviation

with $\geq 53 \%$ remaining free of relapses (Figs. 2a, 3a). Mean changes in EDSS scores did not significantly differ in CARE-MS I patients with HAD treated with SC IFNB-1a $(-0.19)$ versus alemtuzumab $(-0.17)$, but were significantly improved in CARE-MS II HAD patients treated with alemtuzumab $(-0.21)$ versus SC IFNB-1a $(0.16 ; p=0.0351)$. Over 9 years, mean EDSS score remained unchanged in CARE-MS I patients with HAD and increased 0.35 points in CARE-MS II patients with HAD. Sixty-five percent or more of patients with HAD from either CARE-MS I or II achieved stable or improved EDSS scores at year 9 versus core study baseline (Figs. 2b, 3b). In CARE-MS I and II patients with HAD, $91 \%$ of those treated with alemtuzumab were free of 6-month CDW over years 0-2 versus $85-90 \%$ of those treated with SC IFNB-1a, whereas $34-36 \%$ versus $19-32 \%$ achieved CDI over years $0-2$, respectively. Through year 9 , $\geq 55 \%$ of CARE-MS patients with HAD treated with alemtuzumab were free of 6-month CDW, and $\geq 49 \%$ achieved 6-month CDI (Figs. 2c, d, 3c, d).
In year 2, significantly more alemtuzumab-treated patients with HAD were free of MRI disease activity than those treated with SC IFNB-1a $(p \leq 0.0007)$. Over $60 \%$ of patients with HAD treated with alemtuzumab were free of MRI disease activity in each of years 3-9 (Figs. 4a, 5a). More alemtuzumab-treated versus SC IFNB-1a-treated patients with HAD achieved annual NEDA during year 2 in both CARE-MS I and II, and $\geq 53 \%$ of alemtuzumabtreated HAD patients attained NEDA in each of years 3-9 (Figs. 4b, 5b). Over years 3-9, 21\% of CARE-MS I and $14 \%$ of CARE-MS II patients with HAD treated with alemtuzumab had sustained NEDA. Cumulative median BVL from core study baseline was $2.65 \%$ and $1.68 \%$ for alemtuzumabtreated CARE-MS I and II patients with HAD over 9 years, respectively (Figs. 4c, 5c). 

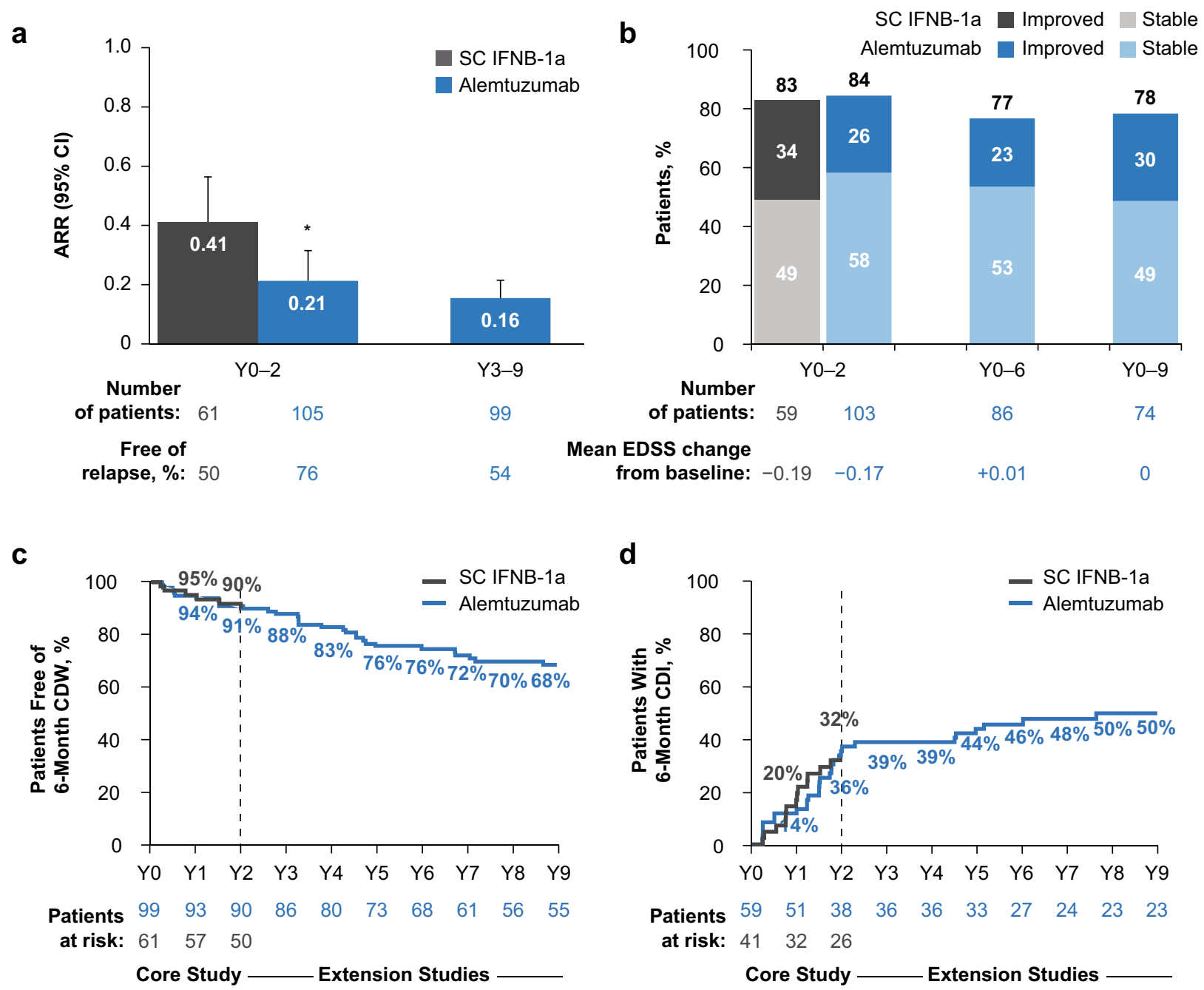

Fig. 2 Clinical efficacy in CARE-MS I alemtuzumab-treated HAD patients through year 9: a ARR; b percentage of patients with improved or stable EDSS scores over time; may not sum appropriately due to rounding; $\mathbf{c}$ percentage of patients free of 6-month CDW; d percentage of patients achieving 6-month CDI. ${ }^{*} p=0.01$ vs. SC IFNB-1a over years $0-2$. Primary HAD definition: $\geq 2$ relapses in the year prior to baseline and $\geq 1$ Gd-enhancing lesion at baseline. Improved EDSS score: $\geq 1$.0-point decrease from core study baseline; stable EDSS score: $\leq 0.5$-point change in either direction from core

\subsection{Sensitivity Analyses of Efficacy: Alternative Definitions of HAD}

To better understand how criteria for HAD of varying restrictiveness affect clinical benefit, sensitivity analyses were carried out using three alternative definitions of HAD applied to the CARE-MS pooled population. Efficacy outcomes for those in the overall pooled CARE-MS I and II population are presented in Supplementary Table 2.

At baseline in the pooled CARE-MS studies, $54 \%$ of alemtuzumab-treated patients satisfied the first alternative definition of HAD (i.e., two or more relapses in year prior

study baseline. CDW: $\geq 1.0$-point EDSS increase (or $\geq 1.5$ points if baseline EDSS $=0$ ) confirmed over 6 months. CDI: $\geq 1$.0-point EDSS decrease from baseline confirmed over 6 months (assessed only in patients with baseline EDSS score $\geq 2.0$ ). ARR annualized relapse rate, CARE-MS Comparison of Alemtuzumab and Rebif ${ }^{\circledR}$ Efficacy in Multiple Sclerosis, CDI confirmed disability improvement, $C D W$ confirmed disability worsening, $C I$ confidence interval, EDSS Expanded Disability Status Scale, $G d$ gadolinium, $H A D$ highly active disease, SC IFNB- $1 a$ subcutaneous interferon- $\beta-1 \mathrm{a}, Y$ year

to baseline, independent of Gd-enhancing lesion count at baseline). Efficacy data were similar between these patients and those meeting the primary HAD definition, except the former had less cumulative BVL over years 0-9 (Table 2).

Of the pooled CARE-MS alemtuzumab patients, $21 \%$ met the second alternative definition of HAD (i.e., at least one relapse in the year prior and three or more Gd-enhancing lesions at baseline) at core study baseline. Efficacy outcomes, specifically ARR, proportions with improved/stable EDSS score, freedom from 6-month CDW, and attainment of 6-month CDI for those who met the second alternative HAD definition were similar to those who met the primary 
a

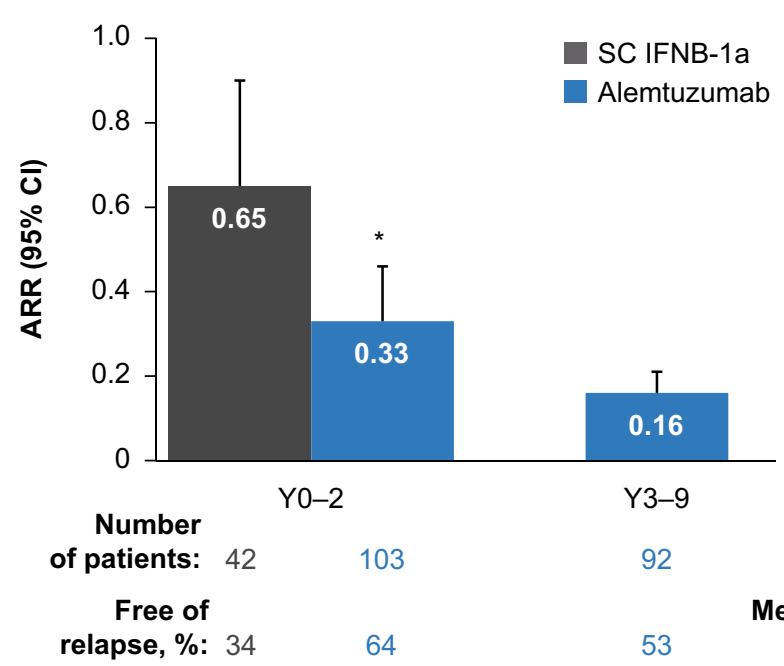

C

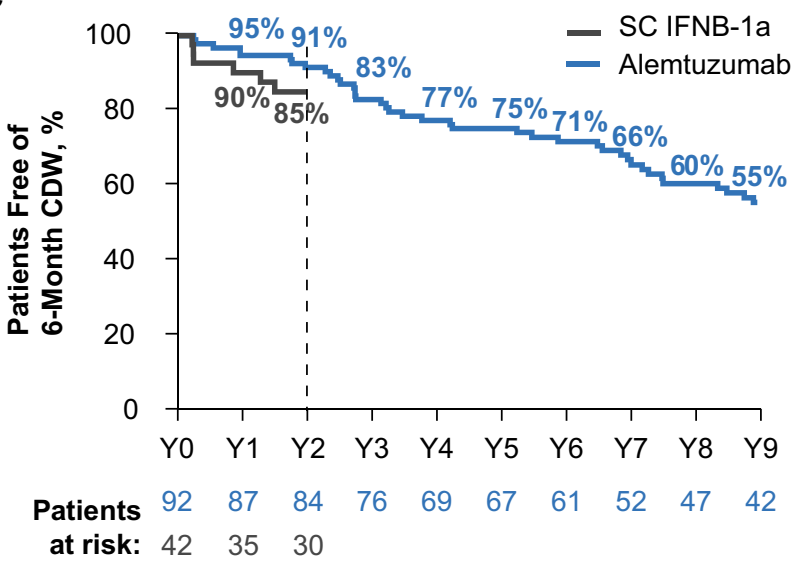

Core Study

Fig. 3 Clinical efficacy in CARE-MS II alemtuzumab-treated HAD patients through year 9: a ARR; b percentage of patients with improved or stable EDSS scores over time; may not sum appropriately due to rounding; $\mathbf{c}$ percentage of patients free of 6-month CDW; d percentage of patients achieving 6-month CDI. $* p=0.004$ vs. SC IFNB-1a over years $0-2$. Primary HAD definition: $\geq 2$ relapses in the year prior to baseline and $\geq 1 \mathrm{Gd}$-enhancing lesion at baseline. Improved EDSS score: $\geq 1.0$-point decrease from core study baseline; stable EDSS score: $\leq 0.5$-point change in either direction from core b

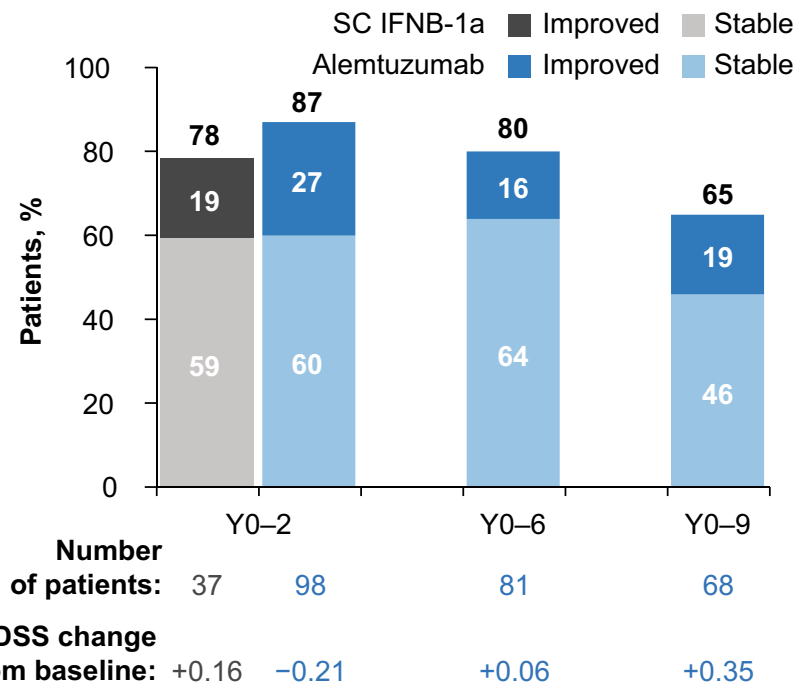

d

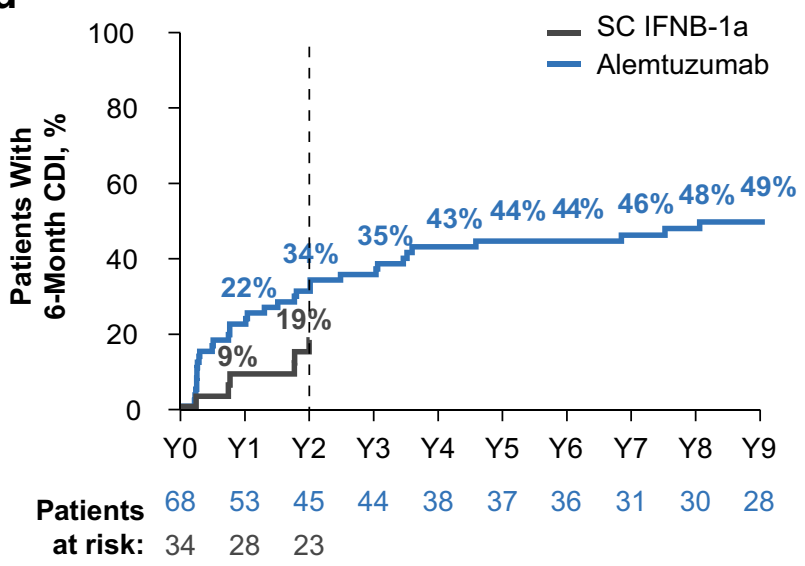

Core Study _ Extension Studies

study baseline. CDW: $\geq 1.0$-point EDSS increase (or $\geq 1.5$ points if baseline EDSS $=0$ ) confirmed over 6 months. CDI: $\geq 1.0$-point EDSS decrease from baseline confirmed over 6 months (assessed only in patients with baseline EDSS score $\geq 2.0$ ). $A R R$ annualized relapse rate, CARE-MS Comparison of Alemtuzumab and Rebif ${ }^{\circledR}$ Efficacy in Multiple Sclerosis, CDI confirmed disability improvement, $C D W$ confirmed disability worsening, $C I$ confidence interval, EDSS Expanded Disability Status Scale, $G d$ gadolinium, $H A D$ highly active disease, SC IFNB- 1 a subcutaneous interferon- $\beta$ - $1 \mathrm{a}, Y$ year

Twenty-two percent of pooled CARE-MS patients met HAD definition. Compared with those who met the primary definition of HAD, patients who met the second alternative definition of HAD experienced a greater EDSS score increase through year 9, were less likely to be relapse-free or to attain sustained NEDA over years 3-9, were less likely to be MRI disease activity-free or to attain annual NEDA during year 9 , and experienced slightly greater cumulative BVL through year 9 (Table 2). the criteria for the third alternative definition of HAD at study baseline (i.e., at least one relapse and at least one Gd-enhancing lesion in the year prior to baseline while on therapy with another DMT). Compared with those who met the primary definition of HAD, those who met the third alternative definition of HAD had similar ARR, attainment of 6-month CDI, freedom from MRI disease activity in 
a

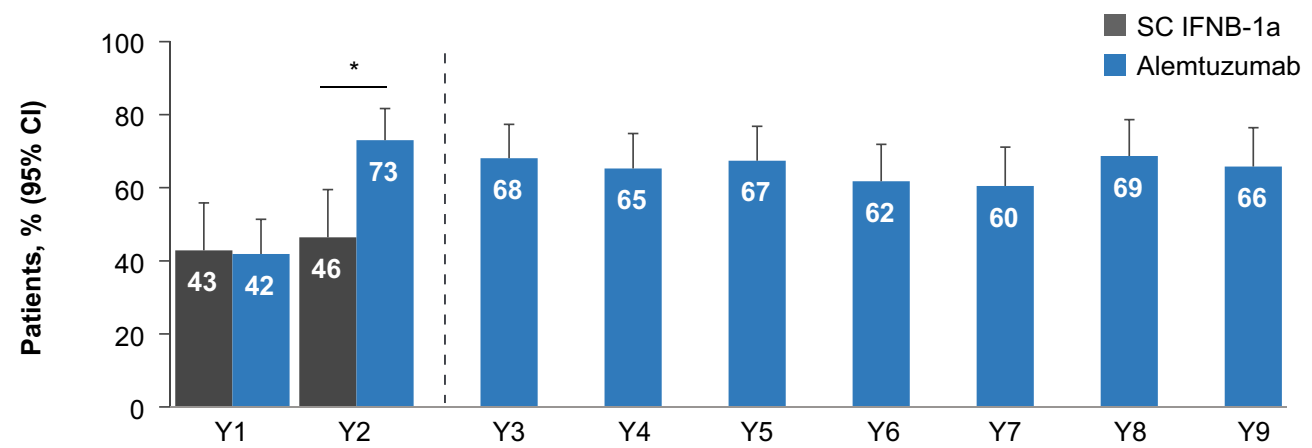

\begin{tabular}{|c|c|c|c|c|c|c|c|c|c|c|c|}
\hline Number of patients: & 56 & 105 & 56 & 100 & 97 & 95 & 95 & 89 & 81 & 83 & 76 \\
\hline \multicolumn{7}{|c|}{ Core Study } & \multicolumn{5}{|c|}{ Extension Studies } \\
\hline $\begin{array}{l}\text { Gd-enhancing } \\
\text { lesion free, \% }\end{array}$ & 84 & 77 & 67 & 91 & 86 & 82 & 82 & 83 & 85 & 84 & 86 \\
\hline $\begin{array}{r}\text { New/enlarging } \\
\text { T2 lesion free, \% }\end{array}$ & 43 & 43 & 46 & 73 & 69 & 66 & 67 & 62 & 60 & 69 & 66 \\
\hline New T1 lesion free, \% & 57 & 70 & 70 & 91 & 85 & 83 & 84 & 78 & 79 & 83 & 88 \\
\hline
\end{tabular}

b

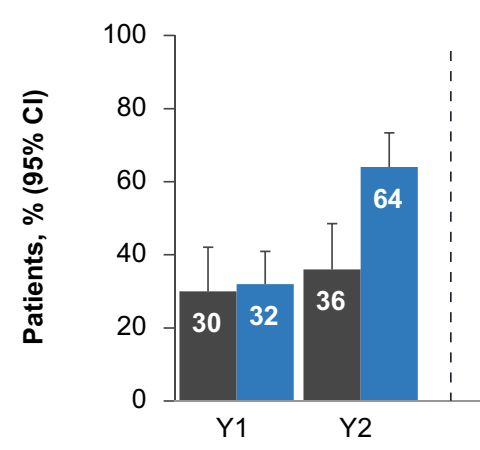

Number of patients: $\quad 56105 \quad 56100$

Core Study

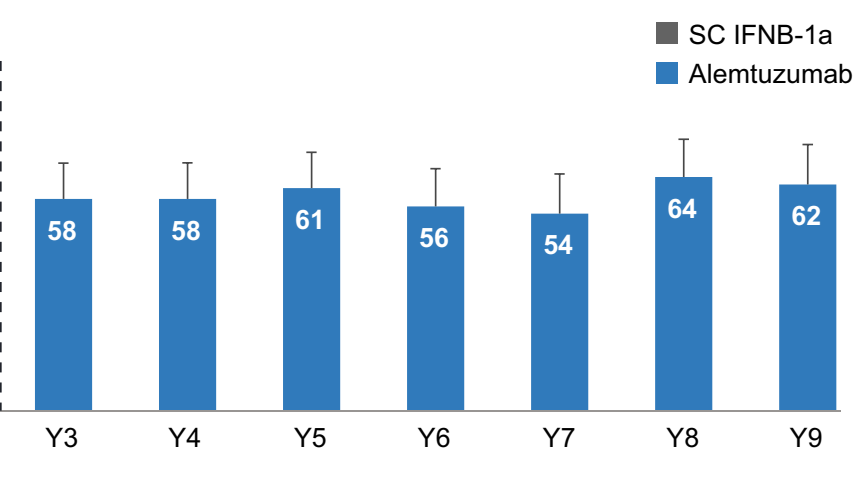

$\begin{array}{lll}97 & 95 & 95\end{array}$

89

$81 \quad 83$

76

C

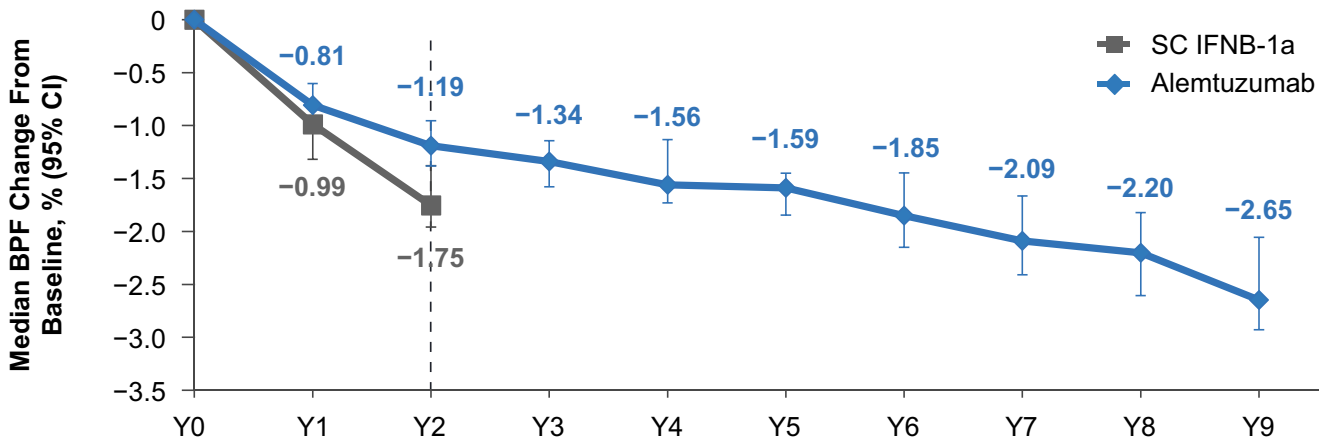

Number of patients:

SC IFNB-1a:

Alemtuzumab: 103

$102 \quad 98$

Core Study

$94 \quad 93$

94

88

80

$81 \quad 75$

Extension Studies 
4Fig. 4 MRI outcomes and NEDA in CARE-MS I alemtuzumabtreated HAD patients through year 9: a percentage of patients free of MRI disease activity; $\mathbf{b}$ percentage of patients achieving annual NEDA; $\mathbf{c}$ cumulative BVL, change from baseline in median BPF over time. $* p=0.0007$ vs. SC IFNB-1a in year 2 . Primary HAD definition: $\geq 2$ relapses in the year prior to baseline and $\geq 1 \mathrm{Gd}$-enhancing lesion at baseline. Freedom from MRI disease activity: no new Gd-enhancing T1 lesions on current MRI and no new/enlarging T2 hyperintense lesions since last MRI. NEDA: absence of relapse, 6-month CDW, and MRI disease activity. $B P F$ brain parenchymal fraction, $B V L$ brain volume loss, CARE-MS Comparison of Alemtuzumab and Rebif ${ }^{\circledR}$ Efficacy in Multiple Sclerosis, $C D W$ confirmed disability worsening, $C I$ confidence interval, $G d$ gadolinium, HAD highly active disease, $M R I$ magnetic resonance imaging, NEDA no evidence of disease activity, $S C$ IFNB-1 a subcutaneous interferon- $\beta-1 \mathrm{a}, Y$ year

year 9, and attainment of NEDA in year 9. Differences in mean EDSS score through year 9, proportions with stable or improved EDSS scores, proportions of relapse-free over years 3-9, proportions free of 6-month CDW, proportions achieving sustained NEDA, and cumulative BVL through year 9 were observed between those who met the third alternative and the primary definitions of HAD. Of these differentiating endpoints, only BVL was favored in those who met the third alternative definition versus those who met the primary HAD definition (Table 2).

\subsection{Safety: Primary HAD Definition}

Incidences of AEs were similar in alemtuzumab-treated patients who met the primary definition of HAD versus the overall alemtuzumab-treated patient population over years 0-9 (Table 3; Supplementary Table 3). In alemtuzumabtreated patients who met the primary HAD definition, annual incidences for overall AEs, IARs, and infections (time trend not shown) declined over time; incidences of serious AEs were $\leq 10.2 \%$ and $\leq 12.6 \%$ in any year in CARE-MS I and II patients, respectively. Autoimmune AEs were predominantly thyroid events, with annual incidences peaking in year 3 (CARE-MS I, 13.7\%; CARE-MS II, 19.8\%) and subsequently declining; no thyroid events were reported in year 9. Three cases of ITP occurred in patients meeting the primary HAD definition in CARE-MS I, and none occurred in CARE-MS II $[11,24]$. One case of nephropathy in a patient meeting the primary HAD definition was reported in each of CARE-MS I and II [20]. Malignancies occurred in three CARE-MS I HAD patients and in no CARE-MS II HAD patients $[11,15,25]$. One death in year 8 occurred in a HAD patient from CARE-MS I who died due to an unknown cause approximately 14 months after the last alemtuzumab dose; the patient had a history of acute systolic congestive heart failure [25]. Two deaths occurred in CARE-MS II HAD patients: a motor vehicle accident in year 2 [12] and a death due to atrioventricular block in year 8 [26], approximately
26 months after the last alemtuzumab dose, which was assessed as not related to alemtuzumab.

\section{Discussion}

Frequent relapses and increased lesion counts early in the RRMS disease course are harbingers of rapid disease progression [27, 28], making reduction of early disease activity in HAD patients a clinically important unmet need. Alemtuzumab is recommended by the American Academy of Neurology DMT guidelines for treatment of HAD patients [10]. Furthermore, a putative treatment model for HAD patients advocates as a first step either biologic immunomodulation with alemtuzumab or chemotherapeutic immunosuppression with cladribine, cyclophosphamide, and mitoxantrone [1]. Recent label changes in Europe, which now indicate alemtuzumab for HAD, also highlight the relevance of this treatment as an option for patients with HAD.[9]

Without an available consensus definition of HAD, further evaluation of alemtuzumab based on multiple definitions varying in focus and restrictiveness is needed for this high-risk population. Proposed definitions for highly active MS, previously referred to as "aggressive" MS, have evolved, and have included various combinations of clinical disease markers such as frequent relapses with incomplete recovery, accumulation of early physical/cognitive impairment, and MRI markers of disease activity (e.g., high lesion frequency despite MS therapy, early brain atrophy) [2]. Our primary definition, which has also been referred to as "rapidly evolving severe MS," was used previously in post hoc analyses of highly active patients from large clinical trials of fingolimod and natalizumab, and is one of several definitions specified by the European Medicines Agency for highly active MS phenotypes [6, 8, 22, 23].

Differences in study populations, study design, and follow-up time prevent direct comparison of alemtuzumab treatment effects with other DMTs. Yet, unlike studies with other DMTs, which examined efficacy and safety in HAD patients over 2 years [22, 23, 29], this analysis assessed outcomes with alemtuzumab in HAD patients over 9 years and demonstrated sustained efficacy in two clinical trials of treatment-naive and inadequate response to prior therapy RRMS populations. Efficacy findings were generally consistent between CARE-MS I and II HAD patients, supporting alemtuzumab as an effective option regardless of prior DMT exposure. In the CARE-MS I and II HAD populations, relapse rates were significantly reduced over 2 years with alemtuzumab compared with SC IFNB-1a, and were accompanied by reductions in MRI lesion activity and BVL. Disability outcomes differed between patients with HAD in each of the CARE-MS studies. In CARE-MS I (i.e., treatment-naive), disability outcomes in alemtuzumab-treated 
a

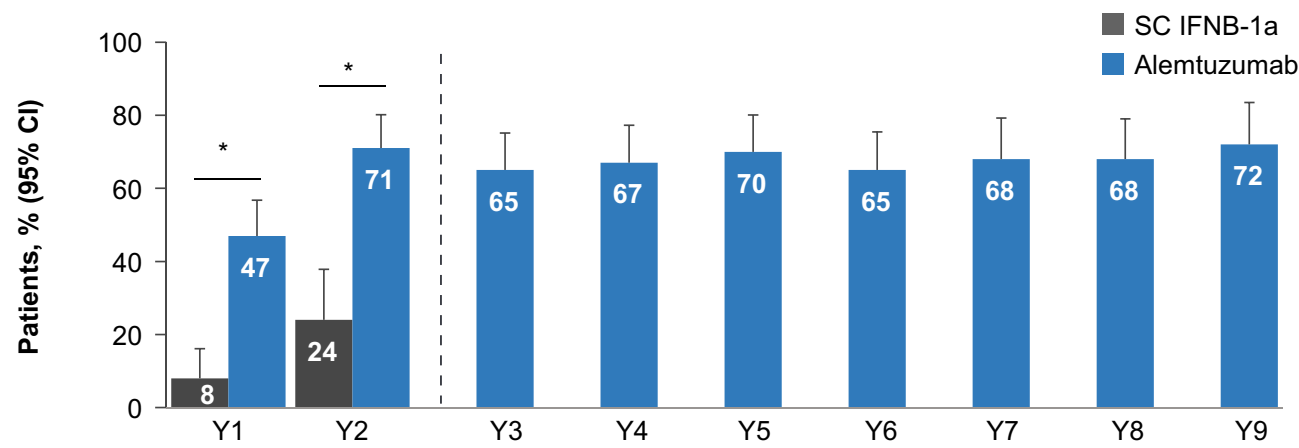

Number of patients: $\quad 40 \quad 101 \quad 37 \quad 94 \quad 85$

Core Study

Gd-enhancing

lesion free, $\%$

$\begin{array}{lllll}65 & 82 & 58 & 87\end{array}$

85

81

80

80

66

$69 \quad 58$

New/enlarging

$\begin{array}{llllllllllll}\text { T2 lesion free, } \% & 8 & 47 & 24 & 71 & 65 & 68 & 70 & 65 & 68 & 69 & 73\end{array}$

$\begin{array}{llllllllllll}\text { New T1 lesion free, } \% & 50 & 76 & 57 & 88 & 85 & 84 & 89 & 90 & 88 & 84 & 93\end{array}$

b

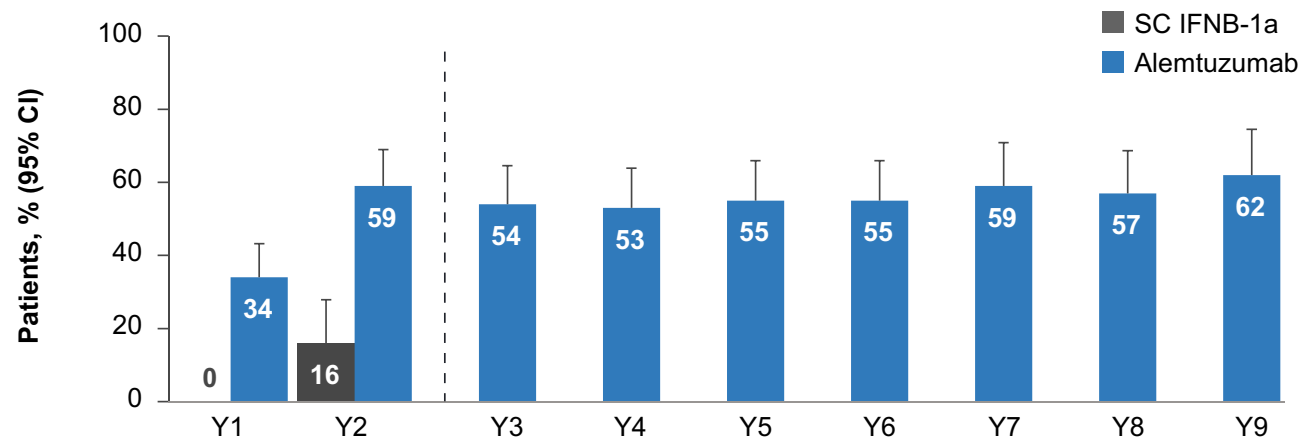

Number of patients: $\quad 40101 \quad 37 \quad 94$

$85 \quad 81 \quad 80$

80

$66 \quad 69 \quad 58$

Core Study

Extension Studies

C

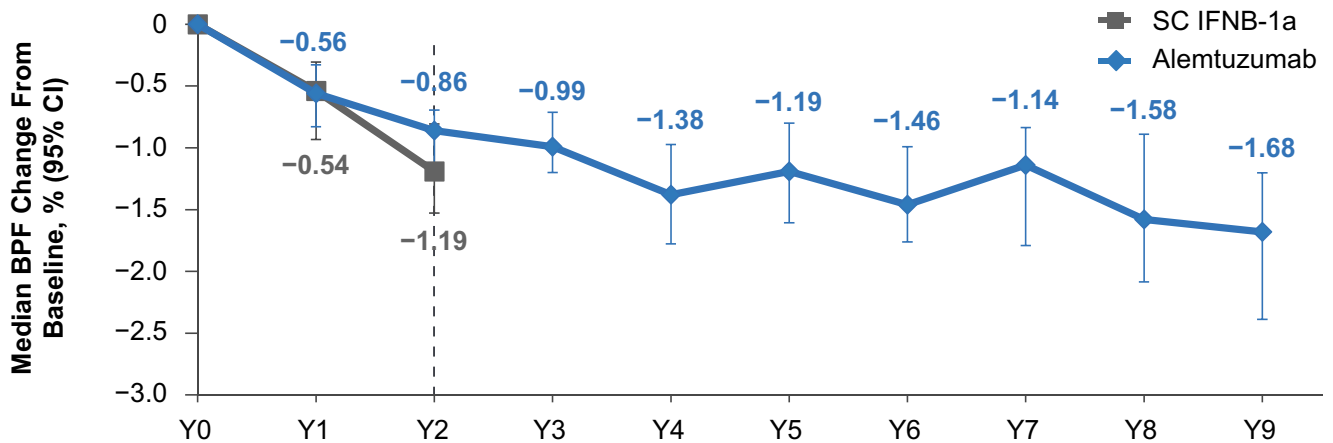

Number of patients:

SC IFNB-1a:

Alemtuzumab: 102

$39 \quad 36$

$100 \quad 95$

Core Study

$87 \quad 81$

$81 \quad 84$

65

69

56

Extension Studies 
4Fig. 5 MRI outcomes and NEDA in CARE-MS II alemtuzumabtreated HAD patients through year 9: a percentage of patients free of MRI disease activity; $\mathbf{b}$ percentage of patients achieving annual NEDA; c cumulative BVL, change from baseline in median BPF over time. ${ }^{*} p<0.0001$ vs. SC IFNB-1a in years 1 and 2 . Primary HAD definition: $\geq 2$ relapses in the year prior to baseline and $\geq 1$ Gd-enhancing lesion at baseline. Freedom from MRI disease activity: no new Gd-enhancing T1 lesions on current MRI and no new/ enlarging T2 hyperintense lesions since last MRI. NEDA: absence of relapse, 6-month CDW, and MRI disease activity. BPF brain parenchymal fraction, $B V L$ brain volume loss, CARE-MS Comparison of Alemtuzumab and Rebif ${ }^{\circledR}$ Efficacy in Multiple Sclerosis, $C D W$ confirmed disability worsening, $C I$ confidence interval, $G d$ gadolinium, $H A D$ highly active disease, $M R I$ magnetic resonance imaging, $N E D A$ no evidence of disease activity, SC IFNB- 1 a subcutaneous interferon$\beta$-1a, $Y$ year

HAD patients were similar to those in SC IFNB-1a-treated patients; whereas, in CARE-MS II (i.e., previously exposed to DMTs), disability outcomes in alemtuzumab-treated HAD patients were superior to those of SC IFNB-1a-treated HAD patients. These outcomes align with previous findings from CARE-MS I, wherein the study was underpowered due to unexpectedly few SC IFNB-1a-treated patients experiencing CDW [11]. No safety signals were unique to the CARE-MS HAD populations over 9 years.

Efficacy results for any of the HAD subgroups analyzed here were generally consistent with those of the overall CARE-MS populations (Supplementary Table 2). Alemtuzumab efficacy was maintained through year 9 in HAD patients, with retention rates and rates of additional alemtuzumab administration similar to the overall CAREMS population. However, BVL was greater in the primary HAD population than in the overall CARE-MS populations (median BPF change for overall alemtuzumab populations over years 0-9: $-2.04 \%$ in CARE-MS I [16] and $-1.22 \%$ in CARE-MS II [14]), potentially due to higher baseline Gd-enhancing lesion burden in the HAD patients. Published evidence has shown the correlation between increased BVL and Gd-enhancing lesion burden in MS patients [30]. In the case of CARE-MS I, the results also indicated stronger disability improvement in the HAD patients treated with alemtuzumab when compared with the overall patient population treated with alemtuzumab, as evidenced by higher attainment of 6-month CDI (50\% vs. $41 \%)$ and a higher proportion of patients with clinically meaningful EDSS score improvement (30\% vs. 20\%) [16]. A recent post hoc analysis of CARE-MS patients through year 6 identified a total of 20 alemtuzumab-treated patients $(3 \%)$ from the overall population who converted to secondary progressive MS (SPMS) after initiating treatment [31]. Of these converting patients, nine met the primary HAD definition at baseline, translating to $4 \%$ of alemtuzumab-treated HAD patients converting to SPMS over 6 years. This low level of SPMS conversion from RRMS among HAD patients further supports the efficacy of alemtuzumab in this high-risk population. These observations in CARE-MS I patients promote the strategy of minimizing damage with aggressive treatment during the critical window of opportunity in the early stages of HAD.

The goals of RRMS treatment continue to evolve with increased availability of high-efficacy agents; the attainment of NEDA has become a realistic possibility in some HAD patients [30, 32]. In this analysis, alemtuzumab treatment resulted in sustained NEDA over years 3-9 in $21 \%$ of CARE-MS I patients and 14\% of CARE-MS II patients with HAD. Studies of DMTs have reported rates of NEDA over similar timeframes for the general MS population, but not specifically in HAD populations, limiting comparisons with this post hoc analysis [32-35].

To account for clinical and MRI contributions toward HAD, additional analyses were carried out on the pooled populations using three alternative definitions. Results observed with the first alternative definition, which had an emphasis on relapse activity in the absence of Gd-enhancing lesions at baseline, were similar to those observed with the primary definition for most endpoints, with the exception of BVL. In patients meeting the first alternative definition, BVL was similar to that in the overall CARE-MS alemtuzumab populations. This likely reflects the substantial proportion of patients free of Gd-enhancing lesions at baseline (52\%) who satisfied the first alternative definition. Because this first alternative definition of HAD accounts only for increased relapse activity, it reveals the treatment benefit in patients with indicators of HAD at early stages based on relapse activity alone. Since these patients may be missed for early intervention due to lack of MRI disease activity, these findings support earlier intervention based on higher relapse rate in order to mitigate disability progression and help minimize brain atrophy. Efficacy of alemtuzumab in patients with either multiple MRI lesions at baseline (i.e., the second alternative HAD definition) or treatment failure in association with clinical and MRI activity (i.e., the third alternative HAD definition) was reduced, with more patients requiring additional alemtuzumab compared with the overall CARE-MS population. Since these two alternative definitions account for contributions of MRI lesions, the data support the argument that earlier intervention with high-efficacy DMT prior to lesion accumulation may render more favorable outcomes.

A limitation of this post hoc analysis was selection bias due to non-stratification of disease activity at randomization. However, in the CARE-MS studies, similar percentages of patients across treatment arms had highly active RRMS, based on the four HAD definitions used here, and retention rates over 9 years were also similar to the overall population. Another limitation was the underpowering of assessments for detection of between-treatment differences for subgroup analyses, which complicated comparisons. 


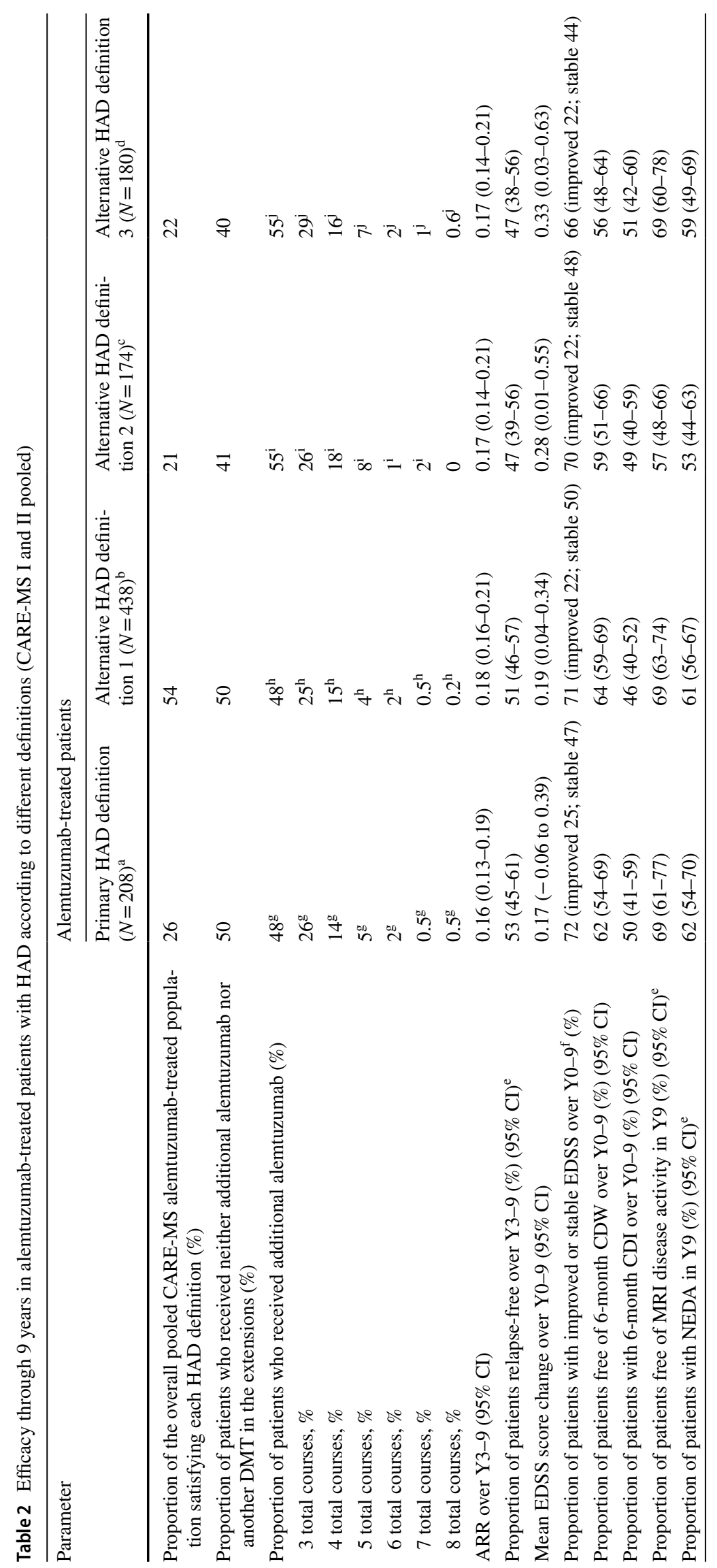




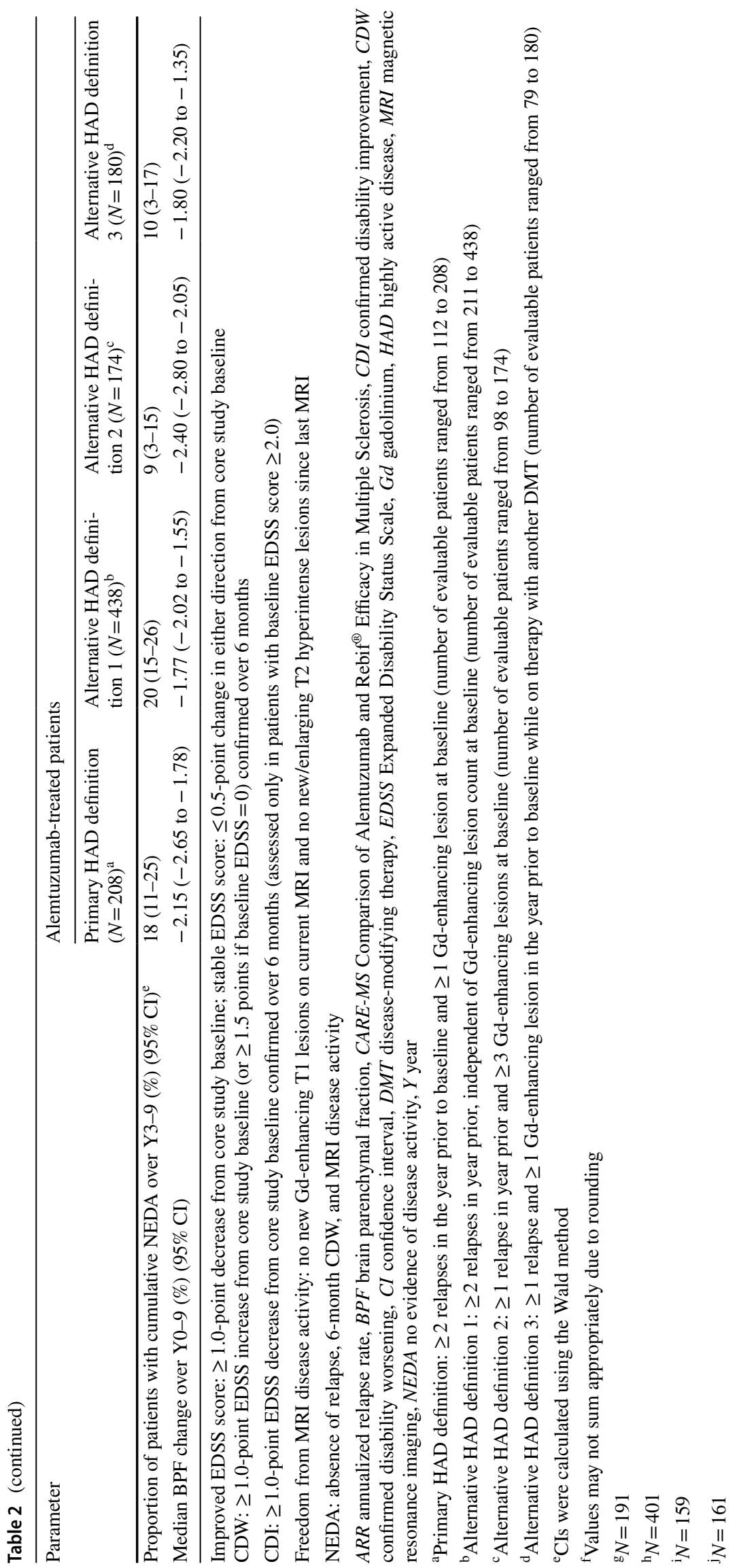


Table 3 AE incidence over years $0-9$ in the HAD alemtuzumabtreated populations from CARE-MS I and II (primary definition)

\begin{tabular}{lll}
\hline AEs, $n(\%)$ & \multicolumn{2}{l}{$\begin{array}{l}\text { Alemtuzumab-treated HAD } \\
\text { patients over years } 0-9\end{array}$} \\
\cline { 2 - 3 } & $\begin{array}{l}\text { CARE-MS I }(N= \\
105)\end{array}$ & $\begin{array}{l}\text { CARE-MS } \\
\text { II }(N= \\
103)\end{array}$ \\
\hline Any AE & $104(99.0)$ & $103(100.0)$ \\
Serious AEs & $40(38.1)$ & $41(39.8)$ \\
Infections & $89(84.8)$ & $93(90.3)$ \\
Serious infections & $7(6.7)$ & $9(8.7)$ \\
Autoimmune AEs ${ }^{\mathrm{a}}$ & & $45(43.7)$ \\
Thyroid AEs & $51(48.6)$ & $7(6.8)$ \\
Serious thyroid AEs & $5(4.8)$ & 0 \\
ITP & $3(2.9)$ & $1(1.0)$ \\
Nephropathies & $1(1.0)$ & 0 \\
Malignancies & $3(2.9)$ & $2(1.9)$ \\
Deaths & $1(1.0)$ &
\end{tabular}

Primary HAD definition: $\geq 2$ relapses in the year prior to baseline and $\geq 1$ Gd-enhancing lesion at baseline

$A E$ adverse event, CARE-MS Comparison of Alemtuzumab and Rebif ${ }^{\circledR}$ Efficacy in Multiple Sclerosis, $G d$ gadolinium, $H A D$ highly active disease, ITP immune thrombocytopenia

${ }^{\text {a }}$ First occurrence of AE within the time period

\section{Conclusions}

These findings demonstrate alemtuzumab is associated with greater improvements in clinical and radiological measures of disease activity compared with SC IFNB-1a over 2 years in patients with highly active RRMS, and may help to control HAD for at least 7 additional years. Efficacy of alemtuzumab in HAD patients was consistent with that seen in the overall CARE-MS population, and there were no safety signals unique to the HAD population in this study.

Acknowledgements Open Access funding provided by Projekt DEAL. The authors and Sanofi thank the patients for their participation in the CARE-MS I, CARE-MS II, CAMMS03409, and TOPAZ studies, as well as the steering committee and the investigators. Critical review of the manuscript for scientific accuracy was provided by Ericka M. Bueno, PhD, and Michael Yeakey, PharmD, of Sanofi. Editorial support was provided by Richard J. Hogan, PhD, and Rebecca L. Orndorff, $\mathrm{PhD}$, of Eloquent Scientific Solutions.

\section{Compliance with Ethical Standards}

Author Contributions TZ, ADB, RB, GC, SE, JH, SFH, CL, VL, DP, CP, SS, LS, AT, BMJU, BVW, and BAS contributed to study design, data collection, drafting and critical review of the manuscript, and approval of the final submission. ZC contributed to drafting and critical review of the manuscript, and approval of the final submission. ND led the statistical support, contributed to the drafting and critical review of the manuscript, and approved the final submission.
Data Availability Qualified researchers may request access to patient level data and related study documents including the clinical study report, study protocol with any amendments, blank case report form, statistical analysis plan, and dataset specifications. Patient level data will be anonymized and study documents will be redacted to protect the privacy of trial participants. Further details on Sanofi's data sharing criteria, eligible studies, and process for requesting access can be found at: https://www.clinicalstudydatarequest.com.

Funding The CARE-MS I, CARE-MS II, CAMMS03409, and TOPAZ studies were funded by Sanofi and Bayer HealthCare Pharmaceuticals. Medical writing support was funded by Sanofi.

Conflict of interest TZ: Consulting and/or speaking fees (Almirall, Bayer, Biogen, Celgene, Merck, Novartis, Roche, Sanofi, and Teva) and grant/research support (Biogen, Novartis, Sanofi, and Teva). ADB: Consulting fees/fees for non-CME services from commercial interests or their agents/grant and research support (Biogen, EMD Serono, Mallinckrodt, Novartis, Roche-Genentech, Sanofi, and TG Therapeutics). RB: Advisory boards and consulting (Acorda, Avanir, Bayer, Biogen, Novartis, Questcor, Sanofi, and Teva). GC: Consulting fees (Actelion, Bayer Schering, Merck Serono, Novartis, Sanofi, and Teva); lecture fees (Bayer Schering, Biogen Dompé, Merck Serono, Novartis, Sanofi, Serono, Symposia International Foundation, and Teva). SE: Speaking and/or consulting (Biogen, Merck Serono, Novartis, Roche, Sanofi, and Teva). JH: Consulting fees, honoraria, travel payment, or research support (Acorda, Bayer-Schering, Biogen, Global Blood Therapeutics, LORA Group, Merck Serono, Novartis, Oxford PharmaGenesis, Roche, Sanofi, Teva, and Vantia) and license fee/royalty payments (Plymouth University). SFH: Consulting agreements, speaker honoraria, writing support, and grant/research financial support (AbbVie, Actelion-Janssen, Adamas, Alkermes, Biogen, Genentech-Roche, Genzyme, Novartis, Osmotica, Mallinckrodt, and Sanofi-Genzyme). CL: Compensation for consulting (Acorda Therapeutics, Bayer, Biogen, Cephalon, EMD Serono, Novartis, Pfizer, Questcor, Sanofi, Strativa, Teva, and UCB). VL: Honoraria for consulting and speaking at symposia (Bayer, Biogen, Merck Serono, Novartis, Roche, Sanofi, and Teva, with approval by the HR Department, Cologne General Hospital, and University of Cologne). DP: Consulting and/or speaking fees and grant/research support (Biogen, Merck Serono, Novartis, Roche, and Sanofi). CP: Consulting and/or speaking fees, research, and travel grants (Actelion, Biogen, Merck, Novartis, Sanofi, and Teva). SS: Consulting and/or speaking fees (Biogen, Merck Serono, Novartis, Sanofi, and Teva) and grant/research support (Novartis and Sanofi). LS: Compensation for advisory board and speaking fees (Bayer, Biogen, Merck Serono, Novartis, Roche, Sanofi, and Teva). AT: Consulting and/or speaking fees and grant/research support (Biogen, Chugai, Roche, Sanofi, and Teva). BMJU: Consulting fees (Biogen, Genzyme, Merck Serono, Novartis, Roche, and Teva). BVW: Research and travel grants, honoraria for MS-expert advice, and speaking fees (Actelion, Bayer-Schering, Biogen, Merck Serono, Novartis, Roche, Sanofi, and Teva). ZC and ND: Employees of Sanofi. BAS: Speaking and/or consulting (AbbVie, Bayer, Biogen, Celgene, EMD Serono, Genentech, Novartis, Roche, Sanofi, Teva, and TG Therapeutics), and research support (AbbVie, Alkermes, Biogen, MedImmune, Novartis, Roche, and Sanofi).

Ethical approval and informed consent Patients provided written informed consent, and all procedures were approved by local institutional ethics review boards of participating sites.

Open Access This article is licensed under a Creative Commons Attribution-NonCommercial 4.0 International License, which permits any non-commercial use, sharing, adaptation, distribution and reproduction 
in any medium or format, as long as you give appropriate credit to the original author(s) and the source, provide a link to the Creative Commons licence, and indicate if changes were made. The images or other third party material in this article are included in the article's Creative Commons licence, unless indicated otherwise in a credit line to the material. If material is not included in the article's Creative Commons licence and your intended use is not permitted by statutory regulation or exceeds the permitted use, you will need to obtain permission directly from the copyright holder. To view a copy of this licence, visit http://creativecommons.org/licenses/by-nc/4.0/.

\section{References}

1. Rush CA, MacLean HJ, Freedman MS. Aggressive multiple sclerosis: proposed definition and treatment algorithm. Nat Rev Neurol. 2015. https://doi.org/10.1038/nrneurol.2015.85.

2. Diaz C, Zarco LA, Rivera DM. Highly active multiple sclerosis: an update. Mult Scler Relat Disord. 2019. https://doi.org/10.1016/j. msard.2019.01.039.

3. Menon S, Shirani A, Zhao Y, Oger J, Traboulsee A, Freedman MS, et al. Characterising aggressive multiple sclerosis. J Neurol Neurosurg Psychiatry. 2013;84(11):1192-8. https://doi. org/10.1136/jnnp-2013-304951.

4. Saccardi R, Freedman MS, Sormani MP, Atkins H, Farge D, Griffith LM, et al. A prospective, randomized, controlled trial of autologous haematopoietic stem cell transplantation for aggressive multiple sclerosis: a position paper. Mult Scler. 2012;18(6):82534. https://doi.org/10.1177/1352458512438454.

5. Biogen Idec. Tysabri ${ }^{\circledR}$ prescribing information and safety update. Available at: https://www.tysabri.com/content/dam/commercial /tysabri/pat/en_us/pdf/tysabri_prescribing_information.pdf. Accessed 26 Nov 2019.

6. Biogen Idec Limited. TYSABRI Summary of Product Characteristics. 2016. http://www.ema.europa.eu/docs/en_GB/document_ library/EPAR_-_Product_Information/human/000603/WC500 044686.pdf. Accessed 8 Jun 2016.

7. Novartis Pharmaceuticals Corporation. US prescribing information: Gilenya ${ }^{\mathrm{TM}}$ (fingolimod) capsules [online]. Available at: http://www.pharma.us.novartis.com/product/pi/pdf/gilen ya.pdf. Accessed 26 Nov 2019.

8. Novartis Europharm Ltd. GILENYA Summary of Product Characteristics. 2015. http://www.ema.europa.eu/docs/en_GB/docum ent_library/EPAR_-_Product_Information/human/002202/ WC500104528.pdf. Accessed 5 Oct 2016.

9. LEMTRADA (alemtuzumab) [Summary of Product Characteristics] January 2020. Diegem, Belgium: Sanofi Belgium. Available at: https://www.medicines.org.uk/emc/medicine/28917. Accessed 1 Feb 2020.

10. Rae-Grant A, Day GS, Marrie RA, Rabinstein A, Cree BAC, Gronseth GS, et al. Practice guideline recommendations summary: Disease-modifying therapies for adults with multiple sclerosis: report of the Guideline Development, Dissemination, and Implementation Subcommittee of the American Academy of Neurology. Neurology. 2018. https://doi.org/10.1212/wnl.00000 00000005347.

11. Cohen JA, Coles AJ, Arnold DL, Confavreux C, Fox EJ, Hartung HP, et al. Alemtuzumab versus interferon beta $1 \mathrm{a}$ as first-line treatment for patients with relapsing-remitting multiple sclerosis: a randomised controlled phase 3 trial. Lancet. 2012. https://doi. org/10.1016/s0140-6736(12)61769-3.

12. Coles AJ, Twyman CL, Arnold DL, Cohen JA, Confavreux C, Fox EJ, et al. Alemtuzumab for patients with relapsing multiple sclerosis after disease-modifying therapy: a randomised controlled phase 3 trial. Lancet. 2012. https://doi.org/10.1016/ s0140-6736(12)61768-1.

13. Coles AJ, Cohen JA, Fox EJ, Giovannoni G, Hartung HP, Havrdova E, et al. Alemtuzumab CARE-MS II 5-year followup: efficacy and safety findings. Neurology. 2017. https://doi. org/10.1212/wnl.0000000000004354.

14. Comi G, Alroughani R, Bass AD, Broadley S, Mao-Draayer Y, Hartung H-P, et al. Alemtuzumab maintains efficacy on clinical and MRI disease activity outcomes, including slowing of brain volume loss, over 9 years in RRMS patients: CARE-MS II followup (TOPAZ study). Mult Scler J. 2019;25(_suppl):P645.

15. Havrdova E, Arnold DL, Cohen JA, Hartung HP, Fox EJ, Giovannoni G, et al. Alemtuzumab CARE-MS I 5-year follow-up: durable efficacy in the absence of continuous MS therapy. Neurology. 2017. https://doi.org/10.1212/wnl.0000000000004313.

16. Montalban X, Arnold DL, Boyko AN, Comi G, Hartung H-P, Kubala Havrdova E, et al. Alemtuzumab maintains efficacy on clinical and MRI disease activity outcomes, including slowing of brain volume loss, over 9 years in RRMS patients: CARE-MS I follow-up (TOPAZ study). Mult Scler J. 2019;25(suppl):P974.

17. Genzyme Corporation. LEMTRADA (alemtuzumab) [Prescribing Information]. Cambridge, MA, USA. Available at: http://products. sanofi.us/Lemtrada/Lemtrada.html. Accessed 26 Nov 2019.

18. Azevedo CJ, Kutz C, Dix A, Boster A, Sanossian N, Kaplan J. Intracerebral haemorrhage during alemtuzumab administration. Lancet Neurol. 2019. https://doi.org/10.1016/s1474 -4422(19)30076-6.

19. Cuker A, Bass AD, Nadj C, Agius MA, Steingo B, Selmaj KW, et al. Immune thrombocytopenia in alemtuzumab-treated MS patients: incidence, detection, and management. Mult Scler. 2019. https://doi.org/10.1177/1352458518816612.

20. Phelps R, Winston JA, Wynn D, Habek M, Hartung HP, Havrdova EK, et al. Incidence, management, and outcomes of autoimmune nephropathies following alemtuzumab treatment in patients with multiple sclerosis. Mult Scler. 2019. https://doi. org/10.1177/1352458519841829.

21. Wray S, Havrdova E, Snydman DR, Arnold DL, Cohen JA, Coles $\mathrm{AJ}$, et al. Infection risk with alemtuzumab decreases over time: pooled analysis of 6-year data from the CAMMS223, CARE-MS I, and CARE-MS II studies and the CAMMS03409 extension study. Mult Scler. 2018. https://doi.org/10.1177/1352458518 796675.

22. Devonshire V, Havrdova E, Radue EW, O’Connor P, Zhang-Auberson L, Agoropoulou C, et al. Relapse and disability outcomes in patients with multiple sclerosis treated with fingolimod: subgroup analyses of the double-blind, randomised, placebo-controlled FREEDOMS study. Lancet Neurol. 2012. https://doi.org/10.1016/ S1474-4422(12)70056-X.

23. Hutchinson M, Kappos L, Calabresi PA, Confavreux C, Giovannoni G, Galetta SL, et al. The efficacy of natalizumab in patients with relapsing multiple sclerosis: subgroup analyses of AFFIRM and SENTINEL. J Neurol. 2009. https://doi.org/10.1007/s0041 5-009-0093-1.

24. Limmroth V, Achiron A, Bass AD, Bertolotto A, Brandes D, Chinea A, et al. Alemtuzumab efficacy and safety were maintained over 8 years in RRMS patients with highly active disease from CARE-MS I: TOPAZ study follow-up. Mult Scler. 2019;25(2_suppl):P1012.

25. Comi G, Arnold DL, Boyko AN, Hartung H-P, Havrdova EK, Inshasi JS, et al. Alemtuzumab improves clinical and MRI disease activity outcomes, including slowing of brain volume loss, in RRMS patients over 8 years: CARE-MS I follow-up (TOPAZ study). Mult Scler. 2018;24(2_suppl):P1235.

26. Singer BA, Alroughani R, Broadley S, Eichau S, Hartung H-P, Havrdova EK, et al. Alemtuzumab improves clinical and MRI disease activity outcomes, including slowing of brain volume loss, 
in RRMS patients over 8 years: CARE-MS II follow-up (TOPAZ study). Mult Scler. 2018;24(2_suppl):P913.

27. Confavreux C, Vukusic S, Adeleine P. Early clinical predictors and progression of irreversible disability in multiple sclerosis: an amnesic process. Brain. 2003;126(Pt 4):770-82.

28. Prosperini L, Gallo V, Petsas N, Borriello G, Pozzilli C. Oneyear MRI scan predicts clinical response to interferon beta in multiple sclerosis. Eur J Neurol. 2009. https://doi.org/10.111 1/j.1468-1331.2009.02708.x.

29. Derfuss T, Bergvall NK, Sfikas N, Tomic DL. Efficacy of fingolimod in patients with highly active relapsing-remitting multiple sclerosis. Curr Med Res Opin. 2015. https://doi. org/10.1185/03007995.2015.1067191.

30. Radue EW, Barkhof F, Kappos L, Sprenger T, Haring DA, de Vera A, et al. Correlation between brain volume loss and clinical and MRI outcomes in multiple sclerosis. Neurology. 2015. https://doi. org/10.1212/wnl.0000000000001281.

31. Horakova D, Boster A, Bertolotto A, Freedman MS, Firmino I, Cavalier SJ, et al. Proportion of alemtuzumab-treated patients converting from relapsing-remitting multiple sclerosis to secondary progressive multiple sclerosis over 6 years. (Submitted).

32. De Stefano N, Stromillo ML, Giorgio A, Battaglini M, Bartolozzi ML, Amato MP, et al. Long-term assessment of no evidence of disease activity in relapsing-remitting MS. Neurology. 2015. https ://doi.org/10.1212/wnl.0000000000002105.

33. Prosperini L, Fanelli F, Pozzilli C. Long-term assessment of no evidence of disease activity with natalizumab in relapsing multiple sclerosis. J Neurol Sci. 2016. https://doi.org/10.1016/j. jns.2016.03.025.

34. Rotstein DL, Healy BC, Malik MT, Chitnis T, Weiner HL. Evaluation of no evidence of disease activity in a 7-year longitudinal multiple sclerosis cohort. JAMA Neurol. 2015. https://doi. org/10.1001/jamaneurol.2014.3537.

35. Uher T, Havrdova E, Sobisek L, Krasensky J, Vaneckova M, Seidl Z, et al. Is no evidence of disease activity an achievable goal in MS patients on intramuscular interferon beta-1a treatment over long-term follow-up? Mult Scler. 2017. https://doi. org/10.1177/1352458516650525.

\section{Affiliations}

Tjalf Ziemssen ${ }^{1}$ - Ann D. Bass ${ }^{2} \cdot$ Regina Berkovich $^{3,4} \cdot$ Giancarlo Comi $^{5} \cdot$ Sara Eichau ${ }^{6} \cdot$ Jeremy Hobart ${ }^{7}$. Samuel F Hunter ${ }^{8}$. Christopher LaGanke ${ }^{9}$. Volker Limmroth ${ }^{10}$. Daniel Pelletier ${ }^{4}$. Carlo Pozzilli ${ }^{11}$. Sven Schippling ${ }^{12} \cdot$ Livia Sousa $^{13} \cdot$ Anthony Traboulsee $^{14} \cdot$ Bernard M. J. Uitdehaag $^{15}$. Bart Van Wijmeersch ${ }^{16}$. Zia Choudhry ${ }^{17}$. Nadia Daizadeh ${ }^{17}$. Barry A. Singer ${ }^{18}$ on behalf of CARE-MS I, CARE-MS II, CAMMS03409, and TOPAZ investigators

Tjalf Ziemssen

Tjalf.Ziemssen@uniklinikum-dresden.de

1 Center of Clinical Neuroscience, University Clinic Carl Gustav Carus, Fetscherstr. 74, 01307 Dresden, Germany

2 Neurology Center of San Antonio, San Antonio, TX, USA

3 Regina Berkovich, MD, PhD, Inc., West Hollywood, CA, USA

4 Keck School of Medicine of University of Southern California, Los Angeles, CA, USA

5 Ospedale San Raffaele, Milan, Italy

6 Hospital Universitario Virgen Macarena, Seville, Spain

7 Plymouth University Peninsula Schools of Medicine and Dentistry, Plymouth, UK

8 Advanced Neurosciences Institute, Franklin, TN, USA

9 North Central Neurology Associates, Cullman, AL, USA

10 Klinik für Neurologie und Palliativmedizin, Cologne, Germany
11 Department of Human Neuroscience, Sapienza University of Rome, Rome, Italy

12 Neuroimmunology and Multiple Sclerosis Research, University Hospital Zürich and University of Zürich, Zürich, Switzerland

13 Centro Hospitalar e Universitário de Coimbra, Coimbra, Portugal

14 University of British Columbia, Vancouver, BC, Canada

15 Amsterdam University Medical Centers, Amsterdam, The Netherlands

16 Rehabilitation and MS-Centre Overpelt, BIOMED, Hasselt University, Hasselt, Belgium

17 Sanofi, Cambridge, MA, USA

18 MS Center for Innovations in Care, Missouri Baptist Medical Center, St. Louis, MO, USA 